\title{
UV coatings by IAD and PARMS technology for Sentinel-5 mission
}

Thomas Weber, Marc Lappschies, Stefan Jakobs, Rik Jansen, James Day, et al.

Thomas Weber, Marc Lappschies, Stefan Jakobs, Rik Jansen, James Day, Ludger van der Laan, Bryan de Goeij, Tim Luijkx, Jeroen Mekking, "UV coatings by IAD and PARMS technology for Sentinel-5 mission," Proc. SPIE 11852, International Conference on Space Optics - ICSO 2020, $118525 Z$ (11 June 2021); doi: 10.1117/12.2600031

SPIE Event: International Conference on Space Optics - ICSO 2021, 2021, Online Only 


\section{International Conference on Space Optics-ICSO 2020}

Virtual Conference

30 March-2 April 2021

Edited by Bruno Cugny, Zoran Sodnik, and Nikos Karafolas
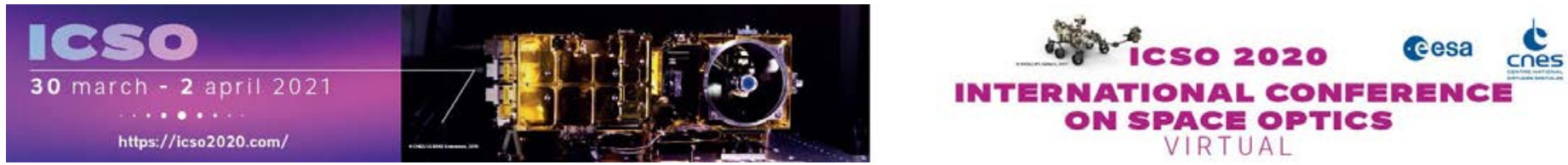

\section{UV coatings by IAD and PARMS technology for Sentinel-5 mission}

\section{Cesa isoporecestings denes}




\title{
UV coatings by IAD and PARMS technology for Sentinel-5 mission
}

\author{
Thomas Weber*a, Marc Lappschies a, Stefan Jakobs ${ }^{\text {a }}$, Rik Jansen ${ }^{\text {b }}$, James Day ${ }^{\text {, }}$, Ludger van der \\ Laan $^{\mathrm{b}}$, Bryan de Goeij ${ }^{\mathrm{b}}$, Tim Luijkx ${ }^{\mathrm{b}}$, Jeroen Mekking ${ }^{\mathrm{b}}$ \\ a Optics Balzers Jena GmbH, Otto-Eppenstein-Straße 2, Jena, Germany, 07745;

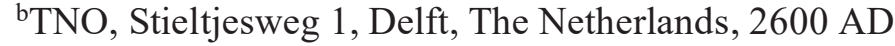

\begin{abstract}
Within the Copernicus program, the Sentinel-5/UVNS instrument is dedicated to the monitoring of air quality, trace gases and aerosols. The instrument consists of two co-aligned telescopes and five spectrometers in the spectral channels named UV1, UV2VIS, NIR, SWIR1, and SWIR3. The spectral band of UV1 spectrometer is defined from $270 \mathrm{~nm}$ to 310 $\mathrm{nm}$. To distribute incoming light and eliminate false light into the channels and within the UV1 channel dedicated coatings for UV spectral range are needed. OBJ was selected for development and application of these coatings.
\end{abstract}

Keywords: coating, PARMS, IAD, UV, mirror, dichroic, evaporation, sputtering

\section{INTRODUCTION}

This presentation will give an overview on different types of UV coatings developed by OBJ for Sentinel-5/UVNS instrument. Two different contributions of Optics Balzers Jena GmbH (OBJ) will be discussed. Firstly, coatings for the telescope beamsplitter optical assembly (TSBOA) and secondly coatings for UV1 spectrometer optics. Sentinel 5 is an ESA project with prime contractor Airbus DS. Manufacturing of TSBOA and UV1 Spectrometer was led by TNO [1,2]. A picture of manufactured PFM models can be found below in figure 1.
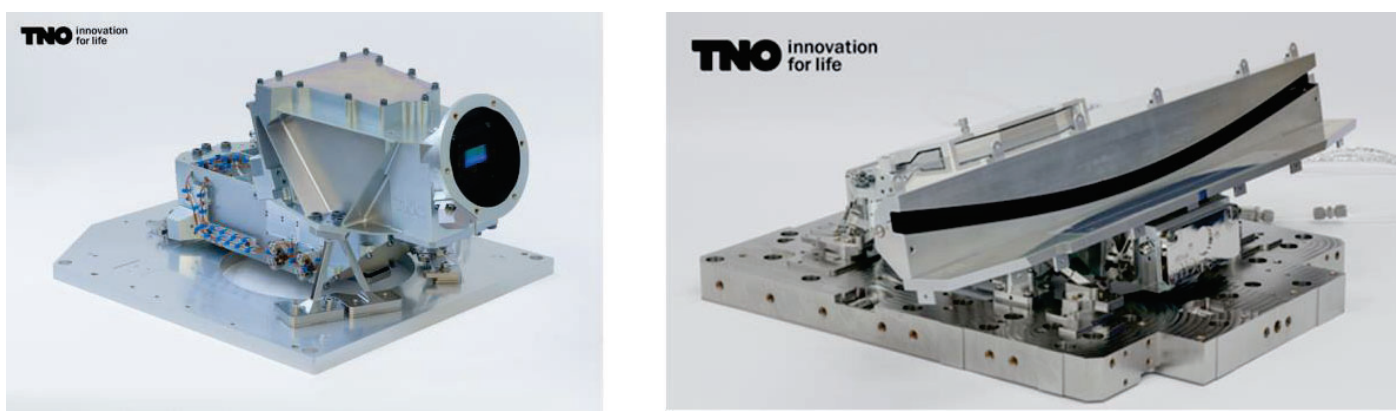

Figure 1. Picture of UV1 spectrometer (left) and TSBOA (right) PFM model. Picture credit TNO.

For both telescope assemblies OBJ was in charge of coating of beam splitters and slit homogenizers inside beam splitter assemblies. In TSBOA VN the incoming light is separated in NIR and UV2VIS channel while TSBOA US splits the light into UV1, SWIR1, and SWIR3 channel. For both, US and VN, beam splitter the UV1 and UV2VIS channels need to be efficiently reflected with specification of $\mathrm{R}>95 \%$. While for $\mathrm{VN}$ beam splitter a layer stack composed of Ta2O5 and $\mathrm{SiO} 2$ is used a third material (HfO2) needed to be added for US beam splitter. Spectral measurements showed a reflectance of above $98.5 \%$ in specified spectral ranges. These coatings were done by means of Plasma Assisted Reactive Magnetron Sputtering (PARMS) [3]. All coating machines at OBJ are equipped with a broad band monitoring system to allow for a precise process control [4].

*thomas.weber@opticsbalzers.com; phone +49 3641 3529411; fax +49 3641 352935; www.opticsbalzers.com 
Reflective coating of homogenizers were done with protected aluminum coatings (Alflex ${ }^{\mathrm{TM}}$ ) by means of an evaporation process. The spectral performance was tuned by the thickness of topmost silica layer and optimized for an AOI range of $86^{\circ}$ to $89^{\circ}$.

Inside the UV1 spectrometer OBJ has manufactured the coating of the five mirrors and the graded coating, as well as the AR coating on the last lens in front of the detector. The mirrors together are specified to follow a specific reflectance template between $270 \mathrm{~nm}$ and $325 \mathrm{~nm}$ and each mirror had a different angle of incidence. So, each mirror had a specific coating design. Beside the reflectance template the set of mirrors was specified with an out of band efficiency of $<$ OD5 in average in the wavelength range from $350 \mathrm{~nm}$ to $1200 \mathrm{~nm}$. The specified spectral performance can only be achieved by a dielectric mirror coating on top of a black coating with low reflectance. To achieve the specified template the design of each mirror of one set needed to be adapted to the already coated mirrors. The black coating was applied by means of magnetron sputtering and the dielectric coatings by means of Ion Assisted Deposition (IAD).

To suppress in-band straylight at the detector a gradient filter was applied on flat surface of the last lens. The gradient filter is a linear variable short pass filter with $\mathrm{T}>90 \%$ and an OD3 blocking level. The gradient was specified in the wavelength range between $250 \mathrm{~nm}$ and $370 \mathrm{~nm}$. It was manufactured by means of IAD technology.

\section{DESIGN AND MANUFACTURING APPROACH}

This chapter briefly describes the driving requirements for each component. Based on that a manufacturing technology was selected to achieve the specified performances.

\subsection{TSBOA beam splitter coatings}

As stated in section 1, the objective of the two telescope beam splitters (VN and US) is to separate the incoming light into the different spectrometer channels with high efficiency. For the $\mathrm{VN}$ beam splitter high reflectance is required in the UV2VIS channel between $300 \mathrm{~nm}$ and $500 \mathrm{~nm}$. High transmittance needs to be achieved in NIR1 and NIR2 bands at 685-710 $\mathrm{nm}$ and 745-773 $\mathrm{nm}$, respectively. Other spectrometer channels are be served by US beam splitter reflecting the UV1 channel (270-310 nm) and transmitting SWIR1 (1590-1675 nm) and SWIR2 (2305-2385 nm) channels.

To achieve the transmittance and reflectance levels of above 98\% PARMS technology is required [5]. For UV reflectance high index material $\mathrm{Ta}_{2} \mathrm{O}_{5}$ is required in the layer stack. As US beam splitter requires reflectance down to 270 $\mathrm{nm} \mathrm{HfO}_{2}$ as third coating material is necessary in the layer stack. PARMS technology allows the deposition of three different materials without interruption of the vacuum chain. On the rear face of the substrate corresponding anti reflective (AR) coatings are applied to allow high transmittance in specified bands and for stress compensation.

\subsection{TSBOA Homogenizer coatings}

In the instrument architecture slit homogenizers are positioned in front of the beam splitter for VN assembly behind the beam splitter (reflected path) for US assembly. So, performance requirements are present for VN homogenizer in UV2VIS, IR1, and IR2 while high reflectance is required for US homogenizer in UV1 channel only. As coating a protected Aluminum mirror, Alflex ${ }^{\mathrm{TM}}$ from Optics Balzers, was selected. Due to the different reflectance ranges, two different coatings are applied. The difference between both coatings is the thickness of the topmost silica layer which can tune the spectral performance in the UV range. Precise control of layer thickness is necessary to achieve the as designed performance. Alflex ${ }^{\mathrm{TM}}$ coatings are applied by e-beam evaporation.

\subsection{UV1 channel mirror coatings}

The UV1 spectrometer consists of five mirrors. Reflectance requirements are specified as combined reflectance for all five mirrors. Each mirror has a different angle of incidence ranging from $8^{\circ}$ to $60^{\circ}$. Beside the spectral performance, the polarization sensitivity had to follow a defined corridor. In this paper, only spectral performance will be discussed. The spectral performance had to comply with the min-max template describing a characteristic spectral shape as shown in figure 2. Figure 2 below also shows the design curves of the five different coatings (left hand side) and the combined 
performance on right hand side. Beside the reflectance profile in the UV1 channel a low reflectance from $340 \mathrm{~nm}$ up to $1200 \mathrm{~nm}$ is required.
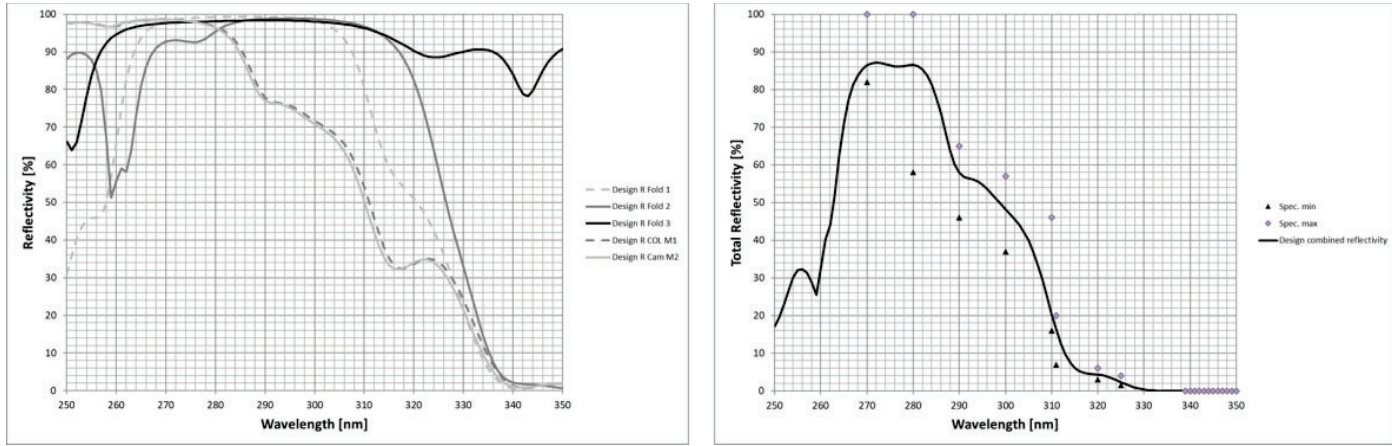

Figure 2. As designed curves of the 5 individual mirror coatings (left) and combined performance versus specification (right).

To achieve these performance two coatings need to be applied. Firstly, a black absorbing coating to generate low reflectance in the long wavelength range and secondly a dielectric mirror coating based on $\mathrm{HfO}_{2}$ to define the reflectance profile of each mirror. As can be seen in figure 2, one mirror has no black coating and only a reflective coating due to design constraints. The approach described above was developed in cooperation between TNO and OBJ.

The black coating based on Chromium was applied by magnetron sputtering. For depositing the dielectric mirror coating IAD technology was used.

\subsection{UV1 channel linear variable filter coating}

To increase the performance of the UV1 spectrometer a linear variable short pass filter is foreseen at the last surface in front of the detector. The coating is applied to the plane back surface of the last lens in the optical system.

Specified coating performance is a short pass coating with transmittance level of above $90 \%$ in UV1 band (270-310 nm) with linear variation of band edge position. The short pass coating is also specified with an OD3 blocking level. The manufacturing was carried out at OBJ by means of Ion Assisted Deposition and the layer stack was composed of an $\mathrm{SiO}_{2} / \mathrm{HfO}_{2}$ multi-layer stack. The same material combination was used for the backside AR coating. Depending on the application linear variable filters can be manufactured by means of IAD or PARMS technology [6].

\section{DISCUSSION OF RESULTS}

In this chapter the results of the flight hardware manufacturing are discussed and compared to the theoretical as designed performance.

\subsection{TSBOA beam splitter coatings}

The beam splitter coatings are carried out by the PARMS process. It can be seen that measured curves follow nicely the as designed curves shown in figures 3 and 4. High reflectance and transmittance is achieved in specified spectral bands. Measurements for US and VN beam splitter are done for angle of incidence $27^{\circ}$ to $34^{\circ}$ and $12^{\circ}$ to $19^{\circ}$, respectively. Spectral curves for minimum and maximum angles are shown. 

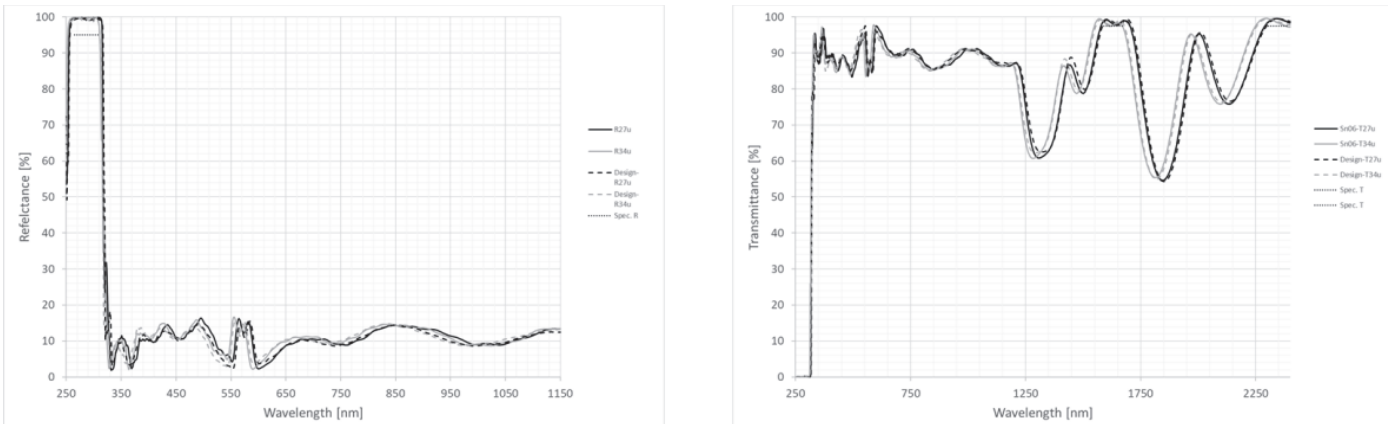

Figure 3. Spectral measurements of US beam splitter; as build performance versus design. Reflectance (left) and transmittance (right) measurements done by spectrophotometry.
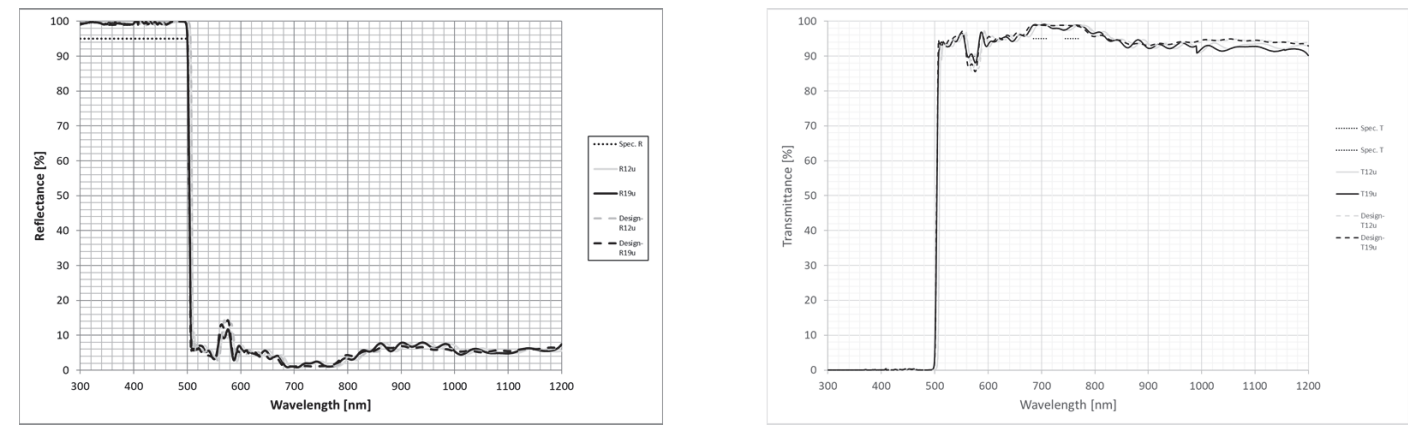

Figure 4. Spectral measurements of VN beam splitter; as build performance versus design. Reflectance (left) and transmittance (right) measurements done by spectrophotometry.

\subsection{TSBOA homogenizer coatings}

The protected aluminum mirror coating, Alflex ${ }^{\mathrm{TM}}$, was manufactured by means of e-beam evaporation. Figure 5 shows the measured reflectance for angles of incidence (AOI) $86^{\circ}, 87^{\circ}, 88^{\circ}$ and $89^{\circ}$. It can be seen that reflectance is very sensitive to AOI. One particular challenge of the homogenizer coating was the performance verification at almost grazing incidence. Spectral measurements with a typical spectrophotometer are not possible. A solution was found by performing the measurements by means of ellipsometry. For this purpose, a particular method was developed at Fraunhofer IOF, Jena. 

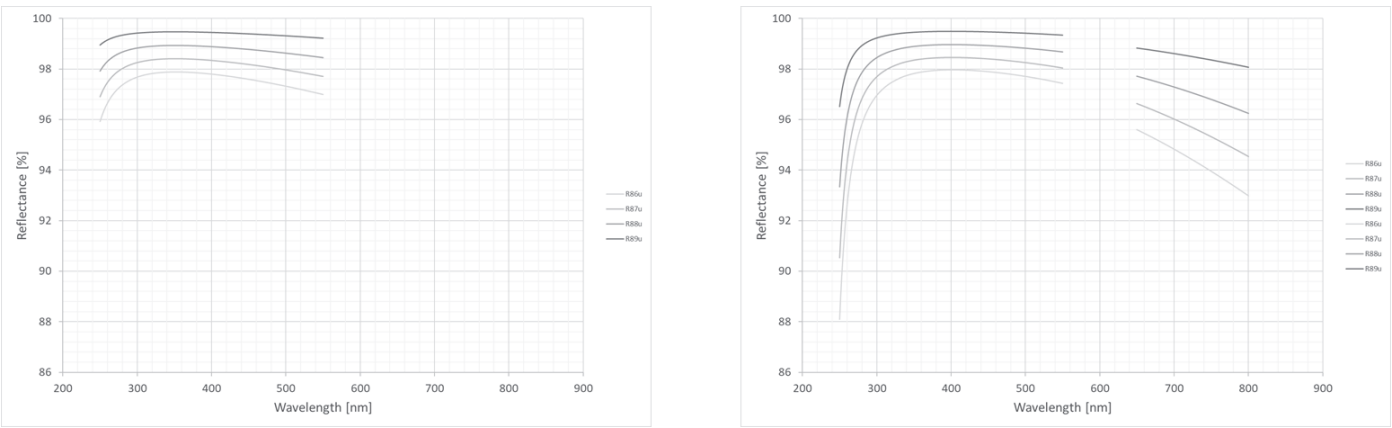

Figure 5. Reflectivity of US (left) and VN (right) homogenizer coating measured by Ellipsometry.

\subsection{UV1 channel mirror coatings}

As the mirror performance is specified as combination of five separate mirrors the manufacturing was quite complex. In a first step, all mirrors were coated with black coatings. Afterwards, it was necessary to test the combined performance of black and dielectric mirror coating by trial coatings. The deposition of dielectric mirror coating on each mirror was done iteratively. After each deposition, the measured result was inserted into a model calculating the combined performance of all five mirrors. This allowed to adapt the following coating in a way to meet the template for combined reflectance.

As the mirrors had a complex geometry, spectral measurements were carried out on witness samples coated together with the mirrors. One performance sample was dedicated to each mirror.

Figure 6 shows the spectral performance in comparison to as designed data. Due to the given envelope and the complex manufacturing approach, the measured combined performance slightly deviates from the design curve but meets the specified template. Beside the reflectance template, also the suppressed reflectance level up to $1200 \mathrm{~nm}$ follows the design prediction.
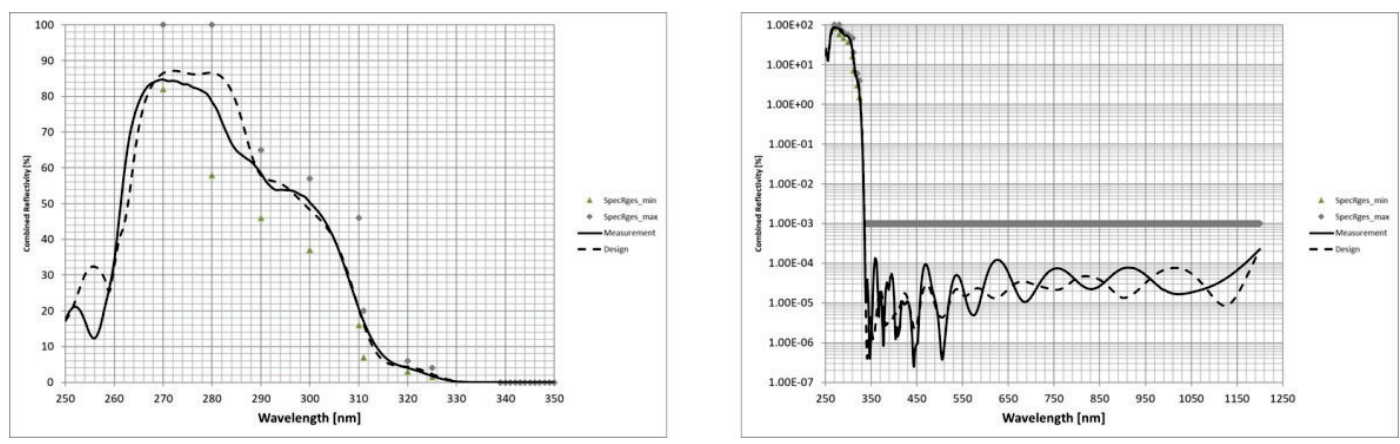

Figure 6. As built performance of combined reflectivity of all 5 mirrors versus template in band (left) and out of ban (right).

\subsection{UV1 channel linear variable filter coating}

Before coating of flight hardware, trial runs on representative substrates were carried out to determine the correct position of the gradient on the substrate. As spectral measurements in transmittance are not possible on the backside of the aspherical lens measurements were done on witness samples coated in the same run. The figure below shows spectral measurements at different positions along the gradient with an achieved transmittance level above $90 \%$ down to $270 \mathrm{~nm}$. 


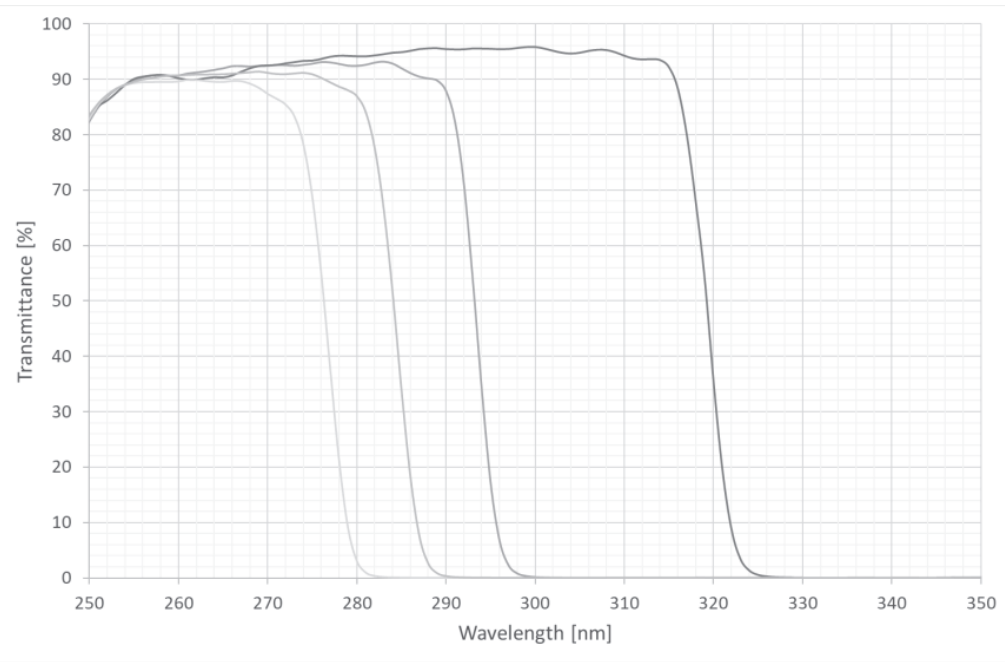

Figure 7. Spectral measurement of gradient filter at different positions along spectral direction.

\section{CONCLUSION}

In this paper, OBJ contribution to Sentinel 5 telescope assembly and UV1 channel optical elements is shown. Different coating technologies are discussed to achieve the specified performances namely e-beam evaporation, ion assisted deposition, magnetron sputtering, and plasma assisted magnetron sputtering. The performance of manufactured mirrors, beam splitters, and linear variable filters was demonstrated. High reflectivity and transmittance down to a wavelength of $270 \mathrm{~nm}$ was achieved for different components.

\section{ACKNOWLEDGEMENTS}

The work presented was done in the framework of Sentinel mission of ESA Copernicus program funded by EU and ESA. OBJ thanks ESA, TNO, and AMOS for selection and cooperation during the development.

\section{REFERENCES}

[1] Irizar, J., Melf, M., Bartsch, P., Koehler, J., Weiss, J., Greinacher, R., Erdmann, M., Kirschner, V., Perez Albinana, A., Martin, D., " Sentinel-5/UVNS," Proc. SPIE 11180, 1118004 (2019).

[2] Jansen, R., Day, J.P.R., Vink, R., de Vreugd, J., van Beekum, E.R.J., van der Laan, L.W., van't Hof, A.C.A., Gielesen, W.L.M., Koehlwer, J., "Design and first light of the Sentinel-5 UV1 spectrometer optics," Proc. SPIE 11151, 111510Q (2019).

[3] Scherer, M., Pistner, J., Lehnert, W., "Innovative production of high quality optical coatings for applications in optics and opto-electronics," 47th Annual Technical Conference Proceedings of the Society of Vacuum Coaters, $179,(2004)$. 
[4] Ristau, D., Ehlers, H., Gross, T., Lappschies, M., "Optical broadband monitoring of conventional and ion processes," Applied Optics, 45, 1495-1501 (2006).

[5] Weber, T., Lappschies, M., Jakobs, S., "Manufacturing of high performance VIS-NIR beam splitters by plasma assisted thin film deposition technologies," Proc. SPIE 10691, 106911A, (2018).

[6] Weber, T., Lappschies, M., Jakobs, S., Meini, M., Gabrieli, R., Taccola, M., "Development of linear variable filters and black coatings by PARMS technology for FLORIS HR focal plane array of FLEX mission," Proc. SPIE 11180, 1118044 (2019). 